\title{
Functional recovery after surgical resection of low grade gliomas in eloquent brain: hypothesis of brain compensation
}

\author{
H Duffau, L Capelle, D Denvil, N Sichez, P Gatignol, M Lopes, M-C Mitchell, J-P Sichez, \\ R Van Effenterre
}

See end of article for authors' affiliations

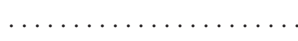

Correspondence to: Dr Duffau, Service de Neurochirurgie, Hôpital de la Salpêtrière, 47-83 Bd de l'hôpital, 75651 Paris, Cedex 13, France; email: hugues.duffau@ psl.ap-hop-paris.fr

\begin{abstract}
Objectives: To describe functional recovery after surgical resection of low grade gliomas (LGG) in eloquent brain areas, and discuss the mechanisms of compensation.

Methods: Seventy-seven right-handed patients without deficit were operated on for a LGG invading primary and/or secondary sensorimotor and/or language areas, as shown anatomically by pre-operative MRI and intraoperatively by electrical brain stimulation and cortico-subcortical mapping. Results: Tumours involved 31 supplementary motor areas, 28 insulas, 8 primary somatosensory areas, 4 primary motor areas, 4 Broca's areas, and 2 left temporal language areas. All patients had immediate post-operative deficits. Recovery occurred within 3 months in all except four cases (definitive morbidity: 5\%). Ninety-two percent of the lesions were either totally or extensively resected on post-operative MRI.

Conclusions: These findings suggest that spatio-temporal functional re-organisation is possible in peritumoural brain, and that the process is dynamic. The recruitment of compensatory areas with long term perilesional functional reshaping would explain why: before surgery, there is no clinical deficit despite the tumour growth in eloquent regions; immediately after surgery, the occurrence of a deficit, which could be due to the resection of invaded areas participating (but not essential) to the function; and why three months after surgery, almost complete recovery had occurred. This brain plasticity, which decreases the long term risk of surgical morbidity, may be used to extend the limits of surgery in eloquent areas.
\end{abstract}

$\mathrm{R}$ esection of infiltrative tumours such as low grade gliomas (LGG) that are located in eloquent brain areas remains a neurosurgical challenge. Although many studies show that the quality of life and the median survival in LGG seems correlated to the extent of resection, ${ }^{1-6}$ the role of resection remains unproven. ${ }^{3}$ Additionally, resection of these tumours, with no clearly defined boundaries and often occurring in functional regions, carries a high risk (13-19.7\%) of permanent deficit. ${ }^{7-9}$ The development of methods of pre-operative neurofunctional imaging (NFI) $)^{10-14}$ and intra-operative electrical stimulations (IES) ${ }^{215-21}$ functional mapping has allowed the identification and the organisation of specific brain regions. These techniques can be used to decrease the risk of complications following surgical resection of tumours in eloquent brain. However, if brain functions are considered to remain in their classical anatomical locations, and these locations are infiltrated by tumour, then excision of the tumour will either be considered impossible or result in an unacceptable postoperative morbidity. ${ }^{19}$ Given the brain plasticity and functional compensation seen after stroke, ${ }^{22}{ }^{23}$ with congenital malformations ${ }^{24} 25$ or following brain injury, we hypothesise that brain infiltration by gliomas leads to reshaping or local reorganisation of functional networks. This would explain the frequent lack of clinical deficit despite LGG growing in eloquent brain areas, ${ }^{27-29}$ and also the transient nature of postoperative deficits. To illustrate the background to this hypothesis we describe our experience and observations of functional recovery in patients having resection of LGG from eloquent brain regions.

\section{PATIENTS AND METHODS}

Between November 1996 and June 2001, a consecutive series of 77 patients had resection of LGG, with IES, from secondary and/or primary functional brain areas. This series comprises 37 males and 40 females (mean age 37 years) who all presented with seizures and a normal neurological examination (except a mild dysphasia in three cases). A neuropsychological evaluation using a standardised questionnaire ${ }^{30}$ showed that all the patients were right handed.

Pre-operative MRI showed a tumour involving the supplementary motor area (SMA) in 31 cases (9 right, 22 left), the insular lobe in 28 cases ( 20 right, 8 left), the primary somatosensory area ( $\mathrm{S} 1)$ in eight cases ( 5 right, 3 left), the primary motor area $(\mathrm{Ml})$ in four cases (three non-dominant facial regions - in one case with also infiltration of S1 of the faceand one hand/forearm left site), Broca's area in four cases (pars opercularis Brodman Area (BA) 44, pars triangularis (BA 45), and the left dominant temporal lobe in two cases.

All surgical procedures used IES ( $5 \mathrm{~mm}$ spaced tips bipolar electrode, biphasic current with pulse duration of $1 \mathrm{~ms}$, frequency of $60 \mathrm{~Hz}$, intensity of 4 to $18 \mathrm{~mA}$, Ojemann Cortical stimulator, Radionics*). ${ }^{18}$ The patients harboring tumours involving the right supplementary and primary motor areas, and the right insular lobe underwent surgery under general anaesthesia, with cortical and sub-cortical IES allowing the detection of the whole cortico-spinal pathways. ${ }^{31}$ Although the motor mapping definition might be considered as not very accurate under general anaesthesia, particularly in subcortical regions, we have recently shown the effectiveness of such a technique, ${ }^{32}$ without "awake surgery". Conversely, the patients with left and somatosensory tumours were operated under

Abbreviations: IES, electrical stimulations; LGG, low grade gliomas; $\mathrm{NFI}$, neurofunctional imaging; SMA, supplementary motor area 


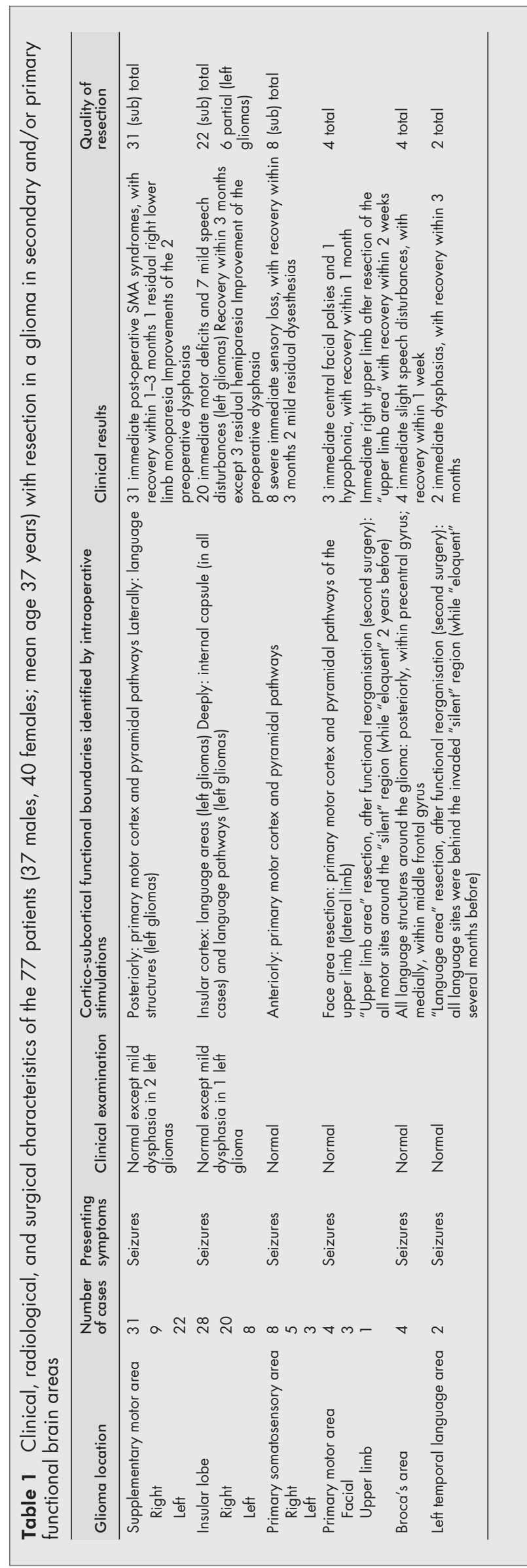

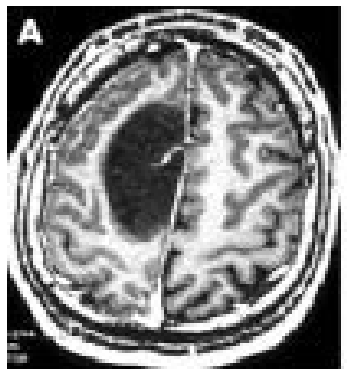
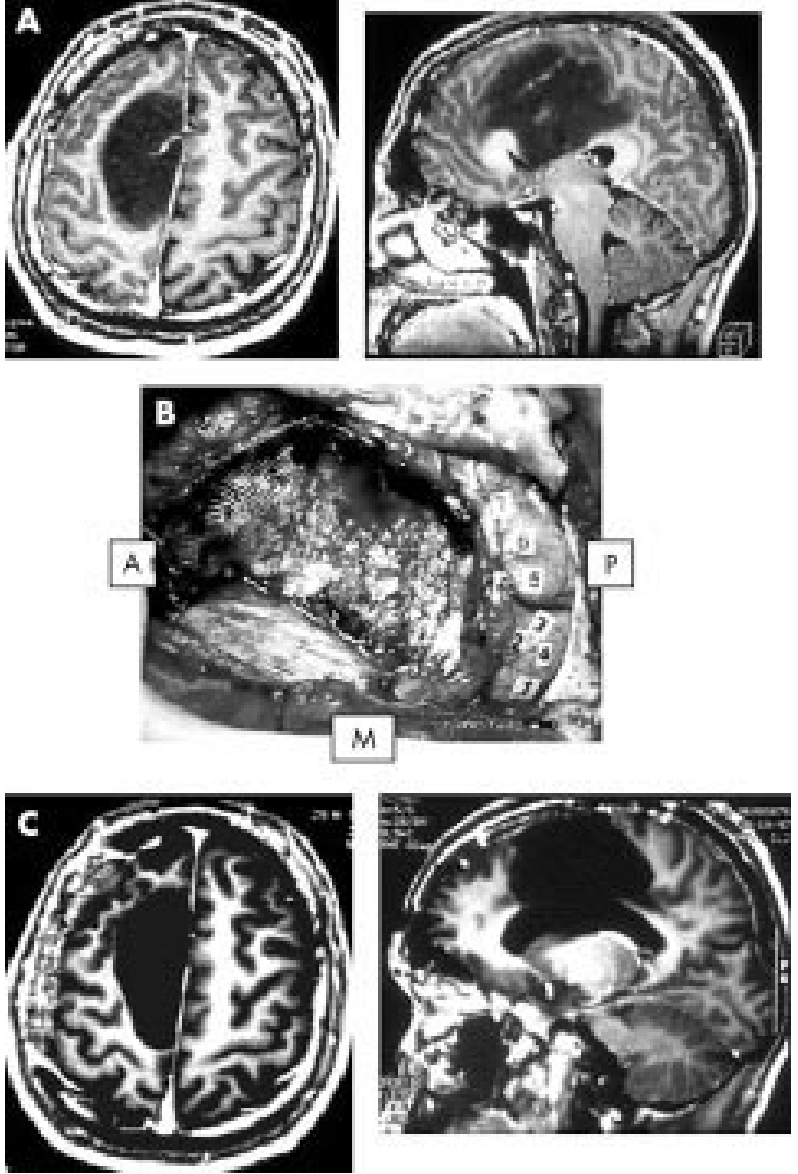

Figure 1 (A) Preoperative axial and sagittal T1-weighted MRI, showing a right medial precentral low grade glioma, involving the supplementary motor area. The neurological examination was normal. (B) Intraoperative photograph after tumour removal. The primary motor cortical sites (marked by numbers from 1-7) and their corresponding descending cortico-spinal pathways, both identified using electrical stimulations, represented the posterior functional boundaries of the resection. $A$, anterior; $P$, posterior; $M$, midline. $(C)$ Postoperative axial and sagittal T1-weighted MRI, showing a total glioma removal, with resection of the supplementary motor area the posterior edge of the surgical cavity comes into the contact with the paracentral area). The patient had an immediate post-surgical hemiplegia, then completely recovered within one month.

local anaesthesia with performance of intraoperative sensorimotor and language mappings. ${ }^{18}$ We never used the technique of extraoperative mapping after placement of subdural grid. Ultrasonography and/or image guidance were used to show the anatomical limits of the tumour. We found the former more useful because of a major brain shift seen during extensive resections.

The 77 patients were examined immediately and three months after the surgery by the same neurologist. An immediate and delayed (three months) post-operative MRI was performed to evaluate the quality of resection.

\section{RESULTS}

The clinical, radiological, and surgical data of the 77 patients are summarised in table 1 . In surgery of medial precentral LGG, the resection was continued posteriorly until the primary motor pathways were detected by cortico-subcortical IES, thus incorporating total SMA removal (fig 1). Laterally, in left LGG, the resection was pursued up to the language structures, notably the head of the caudate nucleus and corresponding white fibres deeply. ${ }^{18}$ No response was elicited 

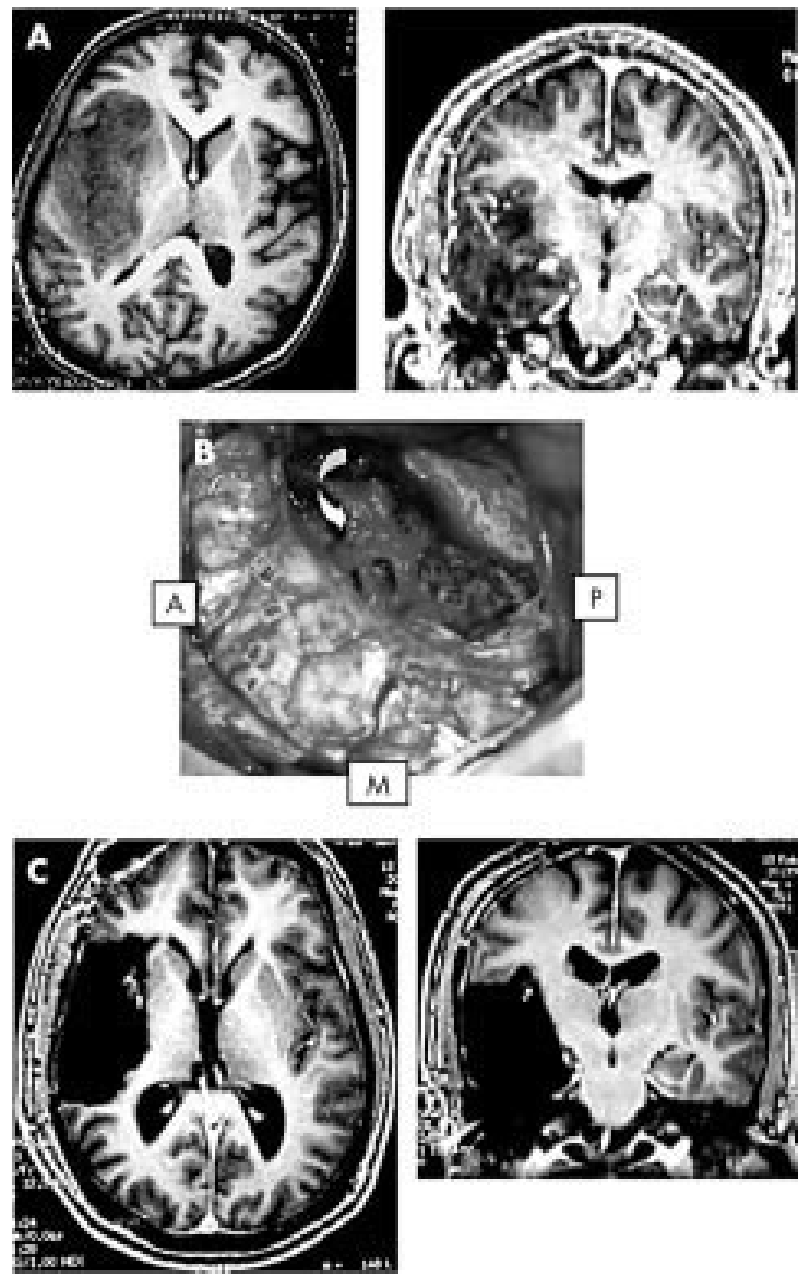

Figure 2 (A) Preoperative axial and coronal T1-weighted MRI, showing a right temporo-insular low grade glioma. The neurological examination was normal. (B) Intraoperative photograph after tumoural removal. The primary motor cortical sites (marked by the tags on the frontal cortex) were preserved, and their corresponding descending cortico-spinal pathways were identified and followed from the corona radiata to the brain stem all along the internal capsule, using electrical stimulations: these motor fibres represented the deep functional boundaries of the resection (numbers 11 and 12 under the sylvian vessels (arrow)). $A$, anterior; $P$, posterior; $M$, midline. (C) Postoperative axial and coronal T1 weighted MRI showing a total glioma removal, with resection of the entire right insular lobe. The patient had an immediate post-surgical hemiparesia, then completely recovered within three months.
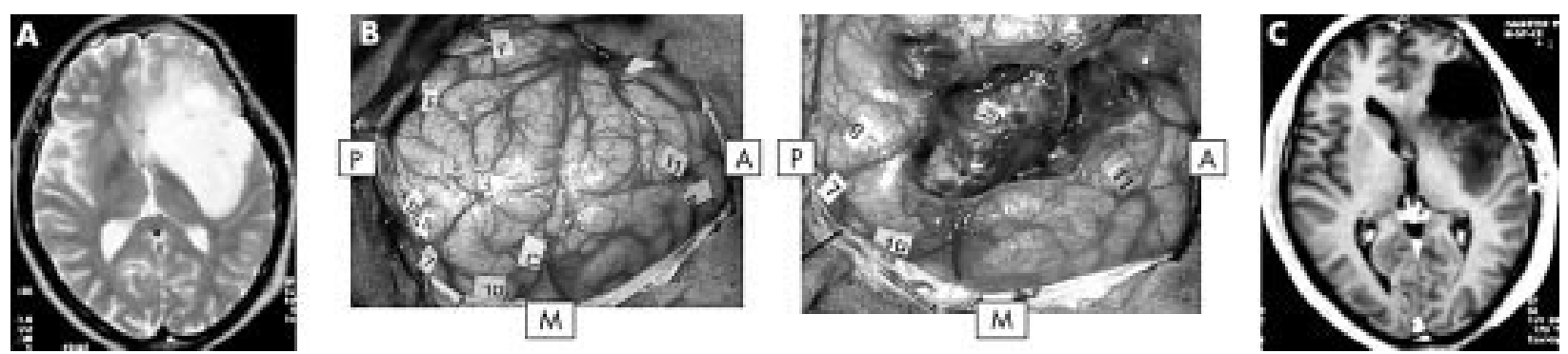

Figure 3 Preoperative axial T2 weighted MRI, showing a left fronto-insular low grade glioma, in the dominant hemisphere. The neurological examination was normal. (B) Left photograph: Intraoperative view before tumour removal. The glioma boundaries were defined using ultrasonography, and marked by letter tags. The eloquent cortical sites identified using electrical stimulations were marked by numbers as follows: 7, primary motor area of the face; 8 , primary somatosensory area of the face; 10 , language site, inducing anarthria during stimulation; 11, language site, inducing speech arrest during stimulation. Right photograph: Intraoperative view after tumour removal. The eloquent cortical sites were preserved $(7,8,10,11)$. However, following the operculum resection, the stimulations of the left dominant insular lobe induced speech arrest (20), leading to stop prematurely the glioma removal. A, anterior; P, posterior; $M$, midline. (C) Postoperative axial T1 weighted MRI, showing a partial glioma removal - that is, with resection of the left frontal operculum, but preservation of the left dominant insular lobe despite its tumoural invasion. The patient had immediate post-surgical speech disturbances, then completely recovered within three months. 

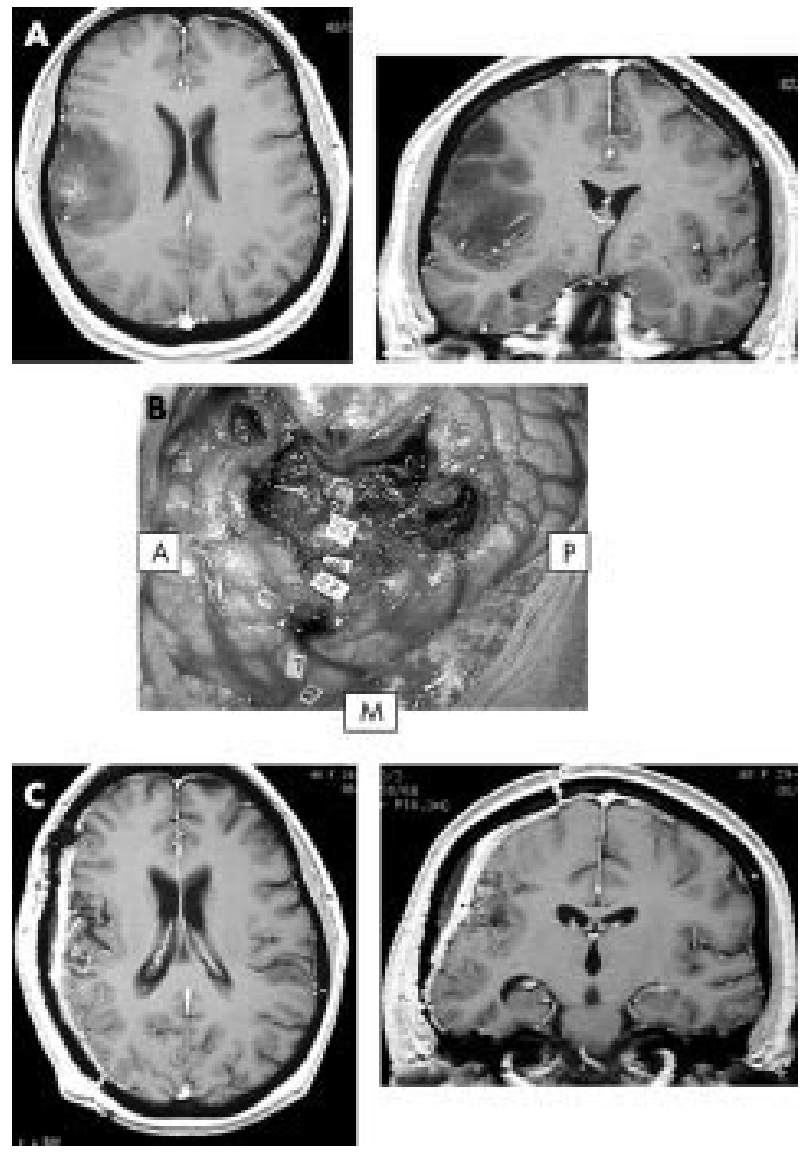

Figure 4 (A) Preoperative axial and coronal T1-weighted MRI, showing a right central supra-sylvian low grade glioma. The neurological examination was normal. (B) Intraoperative photograph after tumour removal. The primary motor cortical sites of the hand (marked by the tags 1 and 2) were preserved, and their corresponding descending cortico-spinal pathways were identified and followed all along the corona radiata $(27,26,25)$, using electrical stimulations. Since these motor fibres of the superior limb represented the deep functional boundaries of the resection, it means that the entire primary sensorimotor area of the face (detected by stimulations within the tumour before its resection) was totally removed. $A$, anterior; $P$, posterior; $M$, midline. (C) Postoperative axial and coronal $\mathrm{Tl}$ weighted MRI, showing a total glioma removal, with resection of the rolandic operculum. The patient had an immediate post-surgical central facial palsy with hypophonia, then completely recovered within one month.

IES) led to cessation of the resection, despite the unmasking of "redundant" motor sites identified by repetitive intraoperative mappings. Two years later, during a second surgery for tumour recurrence, a functional reorganisation of the motor map without any eloquent site within the glioma was observed using IES, and allowed the performance of a total tumoural removal-for example, with resection of a part of the precentral gyrus. The patient had a post-operative monoplegia (right upper limb), but totally recovered within two weeks.

During the removal of the four LGG invading Broca's area (BA 44, BA 45), the limits of resection were represented by the language areas which elicited speech arrest when stimulated, and located around but not within the tumour-that is, in the precentral gyrus, the middle frontal gyrus, and in the pars orbitaris of the inferior frontal gyrus (fig 5). Interestingly, these four patients had only slight transient speech disturbances after surgery, with total recovery within a few days.

Finally, in two left temporal LGG, language (naming) sites were detected within the posterior part of the tumour, leading to incomplete tumour resection. Several months later, during
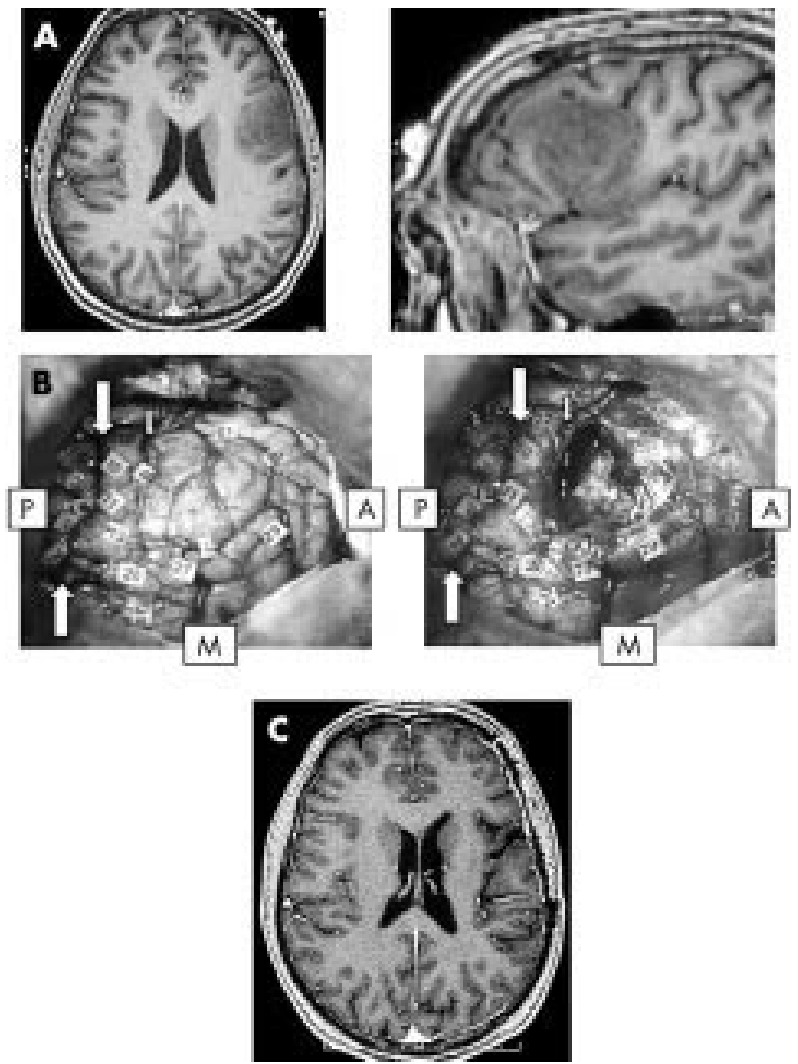

Figure 5 (A) Preoperative axial and sagittal TI weighted MRI, showing a left fronto-opercular low grade glioma (dominant hemisphere), invading pars opercularis (BA 44) and pars triangularis (BA 45) of the inferior frontal gyrus. The neurological examination was normal. (B) Left photograph: Intraoperative view before tumour removal. The glioma boundaries were defined using

ultrasonography, and marked by letter tags $(A, B, C)$. The eloquent cortical sites identified using electrical stimulations were marked by numbers as follows: 1, 2, 3, 5: primary somatosensory area of the face, within the retrocentral gyrus; 10, 11, 13: language sites, inducing anarthria during stimulation, within the precentral gyrus; $26,27,29,34$ : language sites, inducing anomia during stimulation, within the middle frontal gyrus - the more anterior site at the level of the BA 46; 21 : language site, inducing speech arrest during stimulation, in the pars orbitaris of the inferior frontal gyrus (BA 47). No eloquent site was found within the pars opercularis and pars triangularis of the left inferior frontal gyrus, e.g. Broca's area. Right photograph: Intraoperative view after tumour removal. All the eloquent cortical sites were preserved, with resection of the entire pars opercularis and pars triangularis of the left inferior frontal gyrus. $\mathrm{A}$, anterior; $\mathrm{P}$, posterior; $M$, midline; large arrows: central sulcus; small arrow: precentral sulcus. (C) Postoperative axial T1 weighted MRI, showing a total glioma removal, with resection of the Broca's area. The patient had immediate post-surgical slight speech disturbances, then completely recovered within one week.

a second procedure for recurrence, the intraoperative cortical mapping did not find any language area either within or around the tumour, allowing total LGG removal. The patients had a post-operative dysphasia that was more severe than after the first procedure, but recovered in three months.

In summary, all patients had post-operative complete or partial deficits, that recovered in all except four cases (overall definitive morbidity $5 \%$ ). The 73 other patients returned to a normal life.

Postoperative MRI assessment of extent of glioma removal was according to the classification described by Berger et al, ${ }^{1}$ either total (no signal abnormality on T1, T2, and SPGRweighted MRI) or subtotal (less than 10cc of residual tumour) in $92 \%$ (71 patients), and partial in $8 \%$ (six patients) (left insular gliomas). Histological examination confirmed WHO Grade II gliomas in all cases. 


\section{DISCUSSION}

The preoperative findings and postoperative results suggest a dynamic spatio-temporal re-organisation of the focal brain functions can be induced by LGG. This hypothesis would explain:

\section{Preoperative functional compensation:}

The lack of deficit despite a growing tumour in eloquent regions may be explained by the recruitment of compensatory areas-most of the time incorporating local brain sites around the LGG. This is suggested by preoperative NFI. ${ }^{28}{ }^{29}{ }^{33-35}$ We have already reported, using both fMRI and IES, the brain's ability to compensate for speech function in the dominant insula, infiltrated by tumour, by recruiting the left superior temporal gyrus and left putamen. ${ }^{27}$ We have also observed, using IES, a reorganisation of the language sites around the Broca's area (BA 44, BA 45), when invaded by LGG (fig 5 ). Thus, functional compensation is related to recruitment of adjacent regionsfor example, the precentral cortex, the middle frontal gyrus, and the inferior frontal gyrus (pars orbitaris BA 47). Interestingly, these four patients had only slight transient speech disturbances after surgery, with total recovery in only a few days. This transient deficit may be explained by the preoperative reorganisation of language functions. Our data seem in accordance with NFI results, since recent PET data have shown that even limited salvage of peri-infarct tissue after acute stroke treatment can have an important impact on the rehabilitation of cognitive function. ${ }^{36}$ More specifically, Thiel et $\mathrm{al}^{37}$ suggested in cases of brain tumours invaded dominant BA 44 and BA 45 (as in our patients), a functional replacement of these areas by other left fronto-lateral regions such as BA 46 and BA 47.

However, eloquent structures may persist within LGG. Indeed, paresthesias were induced during SI IES despite its tumoural invasion in eight patients, and motor face responses were elicited during MI IES involved by LGG in three patients. Moreover, the speech arrest induced by the left insular cortex IES in six patients, means that a dominant insular lobe entirely infiltrated by a LGG still retains essential language functions (fig 3). Our findings confirm previous reports which detected eloquent areas within tumours (in $7 \%$ to $24 \%$ of cases) using preoperative $\mathrm{NFI}^{38} 39$ or IES. ${ }^{16}{ }^{40}$ These data, likely explained by the Type III spatial configuration of the LGG in the Daumas-Duport's classification, ${ }^{42}$ suggest the persistence within the tumour not only of eloquent neural networks, but also of functional astrocytes and intact neuron-glial interactions. ${ }^{43}{ }^{44}$ Thus, for the neurosurgeon, the difficult question is to know if eloquent functions can be compensated after removal of functioning "brain" tissue, whilst the basic neuroscientist is interested in the mechanisms underlying such compensation.

\section{Intraoperative functional reshaping}

Repetitive IES unmasked several brain regions that performed the same function in patients with LGG involving S1, and Ml of the upper limb. ${ }^{45}{ }^{46}$ These findings show the existence of multiple cortical representations or "functional redundancy" within the primary sensorimotor area, and the human brain's ability to rapidly reorganise its motor map, as seen in animals. ${ }^{47}$ The surgical act itself might induce this reshaping, by generating an hyperexcitability due to changes in the ratio of Gaba:NMDA receptors, ${ }^{45}$ previously described in brain injuries. ${ }^{48-50}$ Indeed, since LGG resection implies removal of functional glio-neuronal structures, this resection might generate changes in neurono-synaptic networks by modulating inhibitory and/or excitatory inputs, as observed using transcranial magnetic stimulations (TMS)..$^{51}$ Thus, we may consider that this short term plasticity can lead to a long term plasticity, as supported by the results of IES in the patients reoperated on several months later, showing modifications of the motor and language maps. These phenomena could explain the postoperative functional recovery. ${ }^{52}$

\section{Postoperative functional compensation}

The main point of this paper is that secondary and even primary eloquent areas may be removed without inducing a definitive deficit. This postsurgical functional compensation could be due to brain plasticity, as in stroke and other injuries. ${ }^{22} 2326$ 53-55 Indeed, the immediate post-operative deficit observed in all patients confirms that some structures remain functional within the tumour mass or peri-tumoural brain, because this deficit is too prolonged to be explained by a simple edema, despite a possible preoperative loco-regional reshaping due to LGG. ${ }^{28}{ }^{29}{ }^{33-35}$ Immediately after surgery, this peritumoural reorganisation is insufficient to maintain function, more so if parts of the "reorganised" brain tissue around the tumour cavity has been transitorily damaged by the surgical trauma itself. Subsequent postoperative development or a reinforcement of this loco-regional mechanism of reshaping and/or the recruitment of remote areas might explain the secondary recovery. ${ }^{56}$ The delay of this recovery (1-3 months) could reflect that neosynaptogenesis, combined with sprouting of axons and dendritis to loco-regional and remote regions, may represent the main plasticity mechanism, as described in vitro and in animals. ${ }^{47} 5758$

On the basis of the NFI results in recovery after stroke, ${ }^{22} 2326$ 53-55 the regions recruited to compensate following resection of eloquent brain invaded by a LGG can be considered for each secondary or primary functional brain area.

\section{SMA resection}

We showed that the occurrence of the SMA syndrome ${ }^{59-61}$ was spatially ${ }^{62}$ and temporally ${ }^{63}$ due to the removal of the SMA proper. ${ }^{64}$ We currently perform postoperative fMRI after SMA syndrome recovery: the preliminary results argue in favour of a compensation by the controlateral SMA, ${ }^{65}$ and also by $\mathrm{Ml}$ (explaining the lack of complete recovery in one patient of our series, due to the associated invasion of the paracentral area).

\section{Insular resection}

We already showed that the left insula, known to play a key role in language, ${ }^{66}$ could be compensated by the left opercula and lentiform nucleus. ${ }^{27}$ This compensation seems possible only when the opercula are not invaded, explaining the total left insular resection in the two LGG type IIIA of Yasargil ${ }^{67}$ but the partial resection in the six other left paralimbic LGG (with invasion of the insula and the opercula-type IIIB of Yasargil). ${ }^{31}$ Concerning the motor function, despite an hemiparesis after insula removal, likely because this region is a non-primary motor area, ${ }^{68}$ all patients recovered (except three due to vascular injury), which could be explained by the recruitment of other non primary motor areas, strongly connected with the insular lobe. ${ }^{68}$

\section{S1 resection}

The first results using pre- and post-operative MEG suggest the possible recruitment of "redundant" eloquent sites around the cavity, within the postcentral gyrus ${ }^{69}$ This is in accordance with the IES data, showing unmasking of redundant somatosensory sites during resection, likely explained by the decrease of the cortico-cortical inhibition. ${ }^{70}$ The recruitment of the second somatosensory area or posterior parietal cortex ${ }^{71} \mathrm{Ml}$ (due to strong anatomo-functional connections between the preand retro-central gyri), ${ }^{45}$ and the controlateral S1 ${ }^{73} 74$ should also be considered.

\section{Resection of non-dominant $\mathrm{M} 1$ of the face}

The recovery of the central facial palsy and hypophonia (already described in opercular syndrome due to cortical or subcortical lesion, ${ }^{75}$ and-in our experience-may be due to 
the resection of both $\mathrm{Ml}$ and $\mathrm{Sl}$ of the face) is likely explained by the disinhibition of the controlateral homologous sites, via the transcallosal pathways. ${ }^{76}$

\section{Resection of $M 1$ of the upper limb}

If we hypothesise the existence of multiple cortical representations, observed in animals, ${ }^{77}$ in humans using fMRI, ${ }^{78}$ and IES, ${ }^{46}$ the compensation of the motor function could be explained by the recruitment of parallel networks within Ml-allowing the superior limb area removal during a second surgery, since the initial rapid reshaping could be durable. ${ }^{79}$

\section{Broca's area resection}

The language compensation may be underlied by the recruitment of adjacent regions (in particular BA46 and BA47). Moreover, Broca's could be not systematically essential for language, as suggested by recent NFI studies in healthy volunteers. ${ }^{80}$

\section{Temporal language area resection}

In the light of fMRI results after recovery from aphasia due to stroke, ${ }^{36}$ language compensation following left dominant temporal resection could be explained by the fact that this function seems to be organised with multiple parallel networks. ${ }^{82}{ }^{83}$ Consequently, beyond the recruitment of areas adjacent to the surgical cavity, the long term reshaping (between two surgeries) could be related to progressive involvement of, firstly, remote regions within the left dominant hemisphere-such as the posterior part of the superior temporal gyrus, ${ }^{84}{ }^{85}$ the triangular part of inferior frontal gyrus ${ }^{86}$ or other left frontolateral regions (BA 46 and BA 47) ${ }^{37}$ and, secondly, the controlateral right non-dominant hemisphere ${ }^{87-89}$ due to a transcallosal disinhibition phenomenon. ${ }^{37}$

However, despite this brain plasticity, mechanisms of compensation are limited, explaining why previous studies of such patients reported irreversible deficits. Firstly, it would seem that reorganisation would be of more importance in secondary than in primary areas. Secondly, if a damaged area is compensated by another region, a lesion of this recruited region will induce a permanent deficit. Consequently, surgical resection should avoid involvement of an eloquent structure if its compensatory area(s) is (are) not perfectly functional (notably if infiltrated by tumour or also removed): this explains why one patient did not completely recover following SMA resection, due to the combined invasion by LGG of Ml of the lower limb (lesion of both secondary and primary eloquent structures).

\section{Authors' affiliations}

H Duffau, L Capelle, D Denvil, M Lopes, J-P Sichez, R Van Effenterre, Department of Neurosurgery, Hôpital Salpêtrière, Paris, France

N Sichez, P Gatignol, Department of Neurology, Hôpital Salpêtrière, Paris, France

M-C Mitchell, Department of Neuroanesthesiology, Hôpital Salpêtrière,

Paris, France

\section{REFERENCES}

1 Berger MS, Deliganis AV, Dobbins JD, et al. The effect of extent of resection on recurrence in patients with low-grade cerebral hemisphere gliomas. Cancer 1994:74:1784-91.

2 Berger MS, Rostomily RC. Low-grade gliomas: Functional mapping resection strategies, extent of resection, and outcome. J Neurooncol 1997;34:85-101.

3 Keles GE, Liamborn KR, Berger MS. Low-grade hemispheric gliomas in adults: a critical review of extent of resection as a factor influencing outcome. J Neurosurg 2001;95:735-45.

4 Nakamura M, Konishi N, Tsunoda S, et al. Analysis of prognostic and survival factors related to treatment of low-grade astrocytoma in adults. Oncology 2000;58:108-16.

5 Piepmeier J, Christopher S, Spencer D, et al. Variations in the natural history and survival of patients with supratentorial low grade astocytomas. Neurosurgery 1996;39:872-79.
6 Scerrati $M$, Roselli $R$, lacoangeli $M$, et al. Prognostic factors in low-grade (WHO grade II) gliomas of the cerebral hemispheres: the role of surgery. J Neurol Neurosurg Psychiatry 1996;61:291-6.

7 Cedzich C, Taniguchi M, Schafer S, et al. Somatosensory evoked potential phase reversal and direct motor cortex stimulation during surgery in and around the central region. Neurosurgery 1996;38:962-70.

8 Fadul C, Wood J, Thaler $\mathrm{H}$, et al. Morbidity and mortality of craniotomy for excision of supratentorial gliomas. Neurology 1988;38:1374-79.

9 Sayawa R, Hammoud M, Schoppa D, et al. Neurological outcomes in a modern series of 400 craniotomies for treatment of parenchymal tumors. Neurosurgery 1998;42:1044-56.

10 Alberstone CD, Skirboll SL, Benzel EC, et al. Magnetic source imaging and brain surgery: presurgical and intraoperative planning in 26 patients. J Neurosurg 2000;92:79-90.

11 Hirsch J, Ruge MI, Kim KHS, et al. An integrated functional magnetic resonance imaging procedure for preoperative mapping of cortical areas associated with tactile, motor, language, and visual functions. Neurosurgery 2000;47:711-22.

12 Hund M, Rezai A, Kronberg E, et al. Magnetoencephalography mapping: basis of a new functional risk profile in the selection of patients with cortical brain lesions. Neurosurgery 1997;40:936-43.

13 Lehéricy S, Duffau H, Cornu P, et al. Correspondence between FMRI somatotopy and individual brain anatomy of the central region: comparison with intrasurgical stimulations in patients with brain tumors. J Neurosurg 2000:92:589-98.

14 McDonald JD, Chong BW, Lewine JD, et al. Integration of preoperative and intraoperative functional brain mapping in a frameless stereotactic environment for lesions near the eloquent cortex. Technical note. J Neurosurg 1999;90:591-98.

15 Berger MS, Ojemann GA. Intraoperative brain mapping techniques in neuro-oncology. Stereotact Funct Neurosurg 1992;58:153-61.

16 Danks RA, Aglio LS, Gugino LD, et al. Craniotomy under local anesthesia and monitored conscious sedation for the resection of tumors involving eloquent cortex. J Neurooncol 2000;49:131-9.

17 Duffau H, Capelle L, Sichez JP, et al. Intraoperative direct electrical stimulations of the central nervous system: the Salpêtrière experience with 60 patients. Acta Neurochir (Wien) 1999;141:1157-67.

18 Duffau H, Capelle L, Sichez N, et al. Intraoperative mapping of the subcortical language pathways using direct stimulations. An anatomo-functional study. Brain 2002;125:199-214.

19 Haglund MM, Berger MS, Shamseldin M, et al. Cortical localization of temporal lobe language sites in patients with gliomas. Neurosurgery 1994;34:567-76

20 Meyer FB, Bates LM, Goerss BS, et al. Awake craniotomy for aggressive resection of primary gliomas located in eloquent brain. Mayo Clin Proc 2001;76:677-87.

21 Taylor MD, Bernstein M. Awake craniotomy with brain mapping as a routine surgical approach to treating patients with supratentorial intraaxial tumors: a prospective trial of 200 cases. J Neurosurg 1999;90:35-41.

22 Chollet $\mathbf{F}$, Weiller $\mathrm{C}$. Imaging recovery of function following brain injury. Curr Opin Neurobiol 1994;4:226-30

23 Weiller C. Imaging recovery from stroke. Exp Brain Res 1998;123:13-17.

24 Lewine JD, Astur RS, Davis LE, et al. Cortical organization in adulthood is modified by neonatal infarct: a case study. Radiology 1994;190:93-6.

25 Vikingstad EM, Cao Y, Thomas $\mathrm{A}$, et al. Language hemispheric dominance in patients with congenital lesions of eloquent brain Neurosurgery 2000;47:562-70.

26 Grady MS, Jane JA, Steward O. Synaptic reorganization within the human central nervous system following injury. J Neurosurg 1989;71:534-37

27 Duffau $\mathbf{H}$, Bauchet L, Lehéricy $\mathrm{S}$, et al. Functional compensation of the left insula dominant for language. Neuroreport 2001:12:2159-63.

28 Fandino J, Kollias SS, Wieser HG, et al. Intraoperative validation of functional magnetic resonance imaging and cortical reorganization patterns in patients with brain tumors involving the primary motor cortex. J Neurosurg 1999;91:238-50.

29 Seitz RJ, Huang Y, Knorr U, et al. Large-scale plasticity of the human motor cortex. Neuroreport 1995;6:742-44.

30 Harris AJ. Harris test of Lateral Dominance. Manual of Directions for Administration and Interpretation. New York: Psychological Corporation 1947

31 Duffau H, Capelle L, Lopes $M$, et al. The insular lobe: physiopathological and surgical considerations. Neurosurgery 2000;47:801-11

32 Duffau H, Capelle L, Denvil D, et al. Usefulness of intraoperative electrical sub-cortical mapping in surgery of low-grade gliomas located within eloquent regions: functional results in a consecutive series of 103 patients. J Neurosurg (in press).

33 Atlas SW, Howard RS, Maldjian J, et al. Functional magnetic resonance imaging of regional brain activity with intracerebral gliomas: findings and implications for clinical management. Neurosurgery 1996:38:329-38

34 Mueller WM, Yetkin FZ, Hammeke TA, et al. Functional magnetic resonance imaging mapping of the motor cortex in patients with cerebral tumors. Neurosurgery 1996;39:515-520.

35 Wunderlich G, Knorr U, Herzog H, et al. Precentral glioma location determines the displacement of cortical hand representation. Neurosurgery 1998;42:18-27. 
36 Warburton E, Price CJ, Swinburn K, et al. Mechanisms of recovery from aphasia: evidence from positron emission tomography studies. J Neurol Neurosurg Psychiatry 1999;66:155-61.

37 Thiel A, Herholz K, Koyuncu A, et al. Plasticity of language networks in patients with brain tumors: a positron emission tomography activation study. Ann Neurol 2001;50:620-9.

38 Ganslandt O, Steimeier R, Kober H, et al. Magnetic source imaging combined with image-guided frameless stereotaxy: a new method in surgery around the motor strip. Neurosurgery 1997;41:621-8.

39 Schiffbauer $\mathbf{H}$, Ferrari $\mathrm{P}$, Rowley $\mathrm{HA}$, et al. Functional activity within brain tumors: a magnetic source imaging study. Neurosurgery 2001;49:1313-21.

40 Ojemann JW, Silbergeld DL. Preserved function in brain involved by tumor. Neurosurgery 1996;39:253-9.

41 Skirboll SS, Ojemann GA, Berger MS, et al. Functional cortex and subcortical white matter located within gliomas. Neurosurgery 1996;38:678-85.

42 Daumas-Duport C, Scheithaver BW, Kelly PJ. A histologic and cytologic method for the spatial definition of gliomas. Mayo Clin Proc 1987:62:435-49.

43 Magistretti PJ, Pellerin L. Cellular mechanisms of brain energy metabolism and their relevance to functional brain imaging. Phil Trans $R$ Soc Lond B 1999;354: 1 155-63.

44 Magistretti PJ. Cellular bases of functional brain imaging: insights from neuron-glia metabolic coupling. Brain Res 2000;886:108-12.

45 Duffau H, Sichez JP, Lehéricy S. Intraoperative unmasking of brain redundant motor sites during resection of a precentral angioma: evidence using direct cortical stimulation. Ann Neurol 2000;47:132-5.

46 Duffau $\mathbf{H}$. Acute functional reorganisation of the human motor cortex during resection of central lesions: a study using intraoperative brain mapping. J Neurol Neurosurg Psychiatry 2001;70:506-13.

47 Sanes JN, Wang J, Donoghue JP. Immediate and delayed changes of rat motor cortical output representation with new forelimb configurations. Cereb Cortex 1992;2:141-52.

48 Eysel UT. Perilesional cortical dysfunction and reorganization. Adv Neurol 1997;73:195-206.

49 Neumann-Haefelin T, Hagemann G, Witte OW. Cellular correlates of neuronal hyperexcitability in the vinicity of photochemically induced cortical infarcts in rats in vivo. Neurosci Lett 1995;193:1-4.

50 Schiene K, Bruehl C, Zilles K, et al. Neuronal hyperexcitability and reduction of GABA receptor expression in the surround of cerebral thrombosis. J Cereb Blood Flow Metab 1996;16:906-914

51 Pascual-Leone A, Tormos JM, Keenan J, et al. Study and modulation of human cortical excitability with transcranial magnetic stimulation. J Clin Neurophysiol 1998;15:333-43.

52 Duffau H, Denvil D, Capelle L. Long term reshaping of language, sensory and motor maps following glioma resection: a new parameter to integrate in the surgical strategy. J Neurol Neurosurg Psychiatry 2002;72:511-6.

53 Cramer SC. Stroke recovery. Lessons from functional MRImaging and other methods of human brain mapping. Phys Med Rehabil Clin N Am 1999; 10:875-86.

54 Imahori $Y$, Fujii R, Kondo $M$, et al. Neural features of recovery from CNS injury revealed by PET in human brain. Neuroreport 1999:10:117-21.

55 Rossini P, Caltagirone C, Castriora-Scanderberg A, et al. Hand motor cortical area reorganization in stroke: a study with fMRI, MEG and TSC maps. Neuroreport 1998;9:2141-6.

56 Rauchecker JP. Mechanisms of compensatory plasticity in the cerebral cortex. Adv Neurol 1997;73:137-46.

57 Bach-Y-Rita P. Brain plasticity as a basis for recovery of function in humans. Neuropsychologia 1990;28:547-54.

58 Jacobs KM, Donoghue JP. Reshaping the cortical motor map by unmasking latent intracortical connections. Science 1991;251:944-7.

59 Laplane D, Talairach J, Meininger V, et al. Clinical consequences of corticectomies involving the Supplementary Motor Area in man. J Neurol Sci 1977;34:301-14

60 Rostomily RC, Berger MS, Ojemann GA, et al. Postoperative deficits and functional recovery following removal of tumors involving the dominant hemisphere supplementary motor area. J Neurosurg 1991;75:62-8

61 Zentner J, Hufnagel A, Pechstein U, et al. Functional results after resective procedures involving the supplementary motor area. J Neurosurg 1996;85:542-9.
62 Fontaine D, Capelle L, Duffau H. The somatotopy of supplementary motor area: evidence by correlation between the extent of surgical resection and the clinical patterns of deficit. Neurosurgery 2002;50:297-305

63 Duffau H, Lopes M, Denvil D, et al. Delayed onset of the supplementary motor area syndrome after surgical resection of the mesial frontal lobe: a time course study using intraoperative mapping in an awake patient. Stereotat Funct Neurosurg 2002;76:74-82

64 Krainik A, Lehéricy S, Duffau $H$, et al. The role of the supplementary motor area in motor deficit following medial frontal lobe surgery. Neurology 2001;57:871-8

65 Krainik A, Lehéricy S, Duffau $\mathrm{H}$, et al. Functional recovery following lesion of the supplementary motor area: a fMRI study. Neuroimage 2001;13:6 (S1206)

66 Dronkers NF. A new brain region for coordinating speech articulation. Nature 1996;384:159-61.

67 Yasargil MG, von Ammon K, Cavazos E, et al. Tumours of the limbic and paralimbic systems. Acta Neurochir (Wien) 1992;1 18:40-52.

68 Augustine JR. Circuitry and functional aspects of the insular lobe in primates including humans. Brain Res Brain Res Rev 1996;22:229-44.

69 Meunier S, Duffau H, Garnero L, et al. Comparison of the somatosensory cortical mapping of the fingers using a whole head magnetoencephalography (MEG) and direct electrical stimulations during surgery in awake patients. Neuroimage 2000;1 1:5 (S868)

70 Duffau H, Capelle L. Récupération fonctionnelle à la suite de lésions de l'aire somato-sensorielle primaire. Etude des mécanismes de compensation. Neurochirurgie 2001;47:557-63.

71 Bittar RG, Olivier A, Sadikot AF, et al. Cortical motor somatosensory representation: effect of cerebral lesions. J Neurosurg 2000;92:242-8.

72 Nii Y, Uematsu S, Lesser RP, et al. Does the central sulcus divide moto and sensory functions? Cortical mapping of human hand areas as revealed by electrical stimulation through subdural grids electrodes. Neurology 1996:46:360-7.

73 Bittar RG, Ptito A, Reutens DC. Somatosensory representation in patients who have undergone hemispherectomy: a functional magnetic resonance imaging study. J Neurosurg 2000;92:45-51.

74 Graveline CJ, Mikulis DJ, Crawley AP, et al. Regionalized sensorimotor plasticity after hemispherectomy fMRI evaluation. Pediatr Neurol 1998; 19:337-42.

75 Posteraro L, Pezzoni F, Varalda E, et al. A case of unilateral opercular syndrome associated with a subcortical lesion. I Neurol 1992;238:337-9.

76 LeRoux PD, Berger MS, Haglund MM, et al. Resection of intrinsic tumors from nondominant face motor cortex using stimulation mapping: report of two cases. Surg Neurol 1991;36:44-48.

77 Donoghue JP, Leibovic S, Sanes JN. Organization of the forelimb area in squirrel monkey motor cortex: representation of digit, wrist and elbow muscles. Exp Brain Res 1992;89: 1-19.

78 Sanes JN, Donoghue JP, Edelman RR, et al. Shared neural substrates controlling hand movements in human motor cortex. Science 1995:268:1775-7.

79 Garraghty PE, Kaas JH. Large-scale functional reorganization in adult monkey cortex after peripheral nerve injury. Proc Natl Acad Sci USA 1991:88:6976-80

80 Etard O, Mellet E, Papathanassiou D, et al. Picture naming without Broca's and Wernicke's area. Neuroreport 2000;11:617-22.

81 Mimura $M$, Kato $M$, Kato $M$, et al. Prospective and retrospective studies of recovery in aphasia. Changes in cerebral blood flow and language functions. Brain 1998:121:2083-94.

82 Mesulam MM. From sensation to cognition. Brain 1998;121:1013-52.

83 Small SL. Connectionist networks and language disorders. J Commun Disord 1994; 27:305-23.

84 Heiss WD, Kessler J, Thiel A, et al. Differential capacity of left and right hemispheric areas for compensation of poststroke aphasia. Ann Neurol 1999:45:419-20.

85 Karbe H, Herholz K, Kessler J, et al. Recovery of language after brain damage. Adv Neurol 1997;73:347-58.

86 Kessler J, Thiel A, Karbe H, et al. Piracetam improves activated blood flow and facilitates rehabilitation of poststroke aphasic patients. Stroke 2000;31:2112-6.

87 Cappa SF, Perani D, Grassi F, et al. A PET follow-up study of recovery after stroke in acute aphasics. Brain Lang 1997;56:55-67.

88 Musso $M$, Weiller C, Kiebel S, et al. Training-induced brain plasticity in aphasia. Brain 1999;122:1781-90.

89 Weiller C, Isensee C, Rijntjes M, et al. Recovery from Wernicke's aphasia: a positron emission tomographic study. Ann Neurol 1995; 37:723-32. 